Gynecol Obstet Invest 1995;40:283-284

\title{
Subject Index Vol. 40, 1995
}

Abortion 108 Aceto-white lesions 265 ACTH 42 Acute pelvic pain 61

- $\quad$ toxoplasma infection 36

Adenocarcinoma 69

Adenylate cyclase inhibition 73

Adult-onset amenorrhea 200

Amenorrhea 42

Amniocentesis 28

Amnion 73 Amniotic fluid 231

- embolism 14

- index 28

- infection 28 Androgens 217 Aphrodisiacs 145

Atrial natriuretic peptide 190

Backup tissue sampling 94 Bartholin's gland duct 278 Birth weight, prediction 253 Bleomycin 57 Body fat 195

CA125 61 Carcinoma 129 Case-referent study 183 Cell cycle 57 Cervical cancer 57

carcinoma 133

dysplasia 52

pregnancy 213 Cesarean section 89 Chemotherapy 213 Chorionic villous sampling 11 Chronic toxoplasma infection 36 Cisplatin 57

Clinical effects 125

Clinically manifest infants 36

Clonality 204

Computerized tomography 215

Continuous-wave Doppler 80

Contractions 11

Controlled ovarian hyperstimulation

117 Cortisol 42 Cyclic guanosine monophosphate 190

Differential methylation 204

- $\quad$ polymerase chain reaction 209

DNAploidy 129

Dopamine antagonist 42

Doppler flow 24

- $\quad$ ultrasound 19, 32

Double-J catheter 274

Drug-induced agranulocytosis 276

Dysplasia 278

Ectopic pregnancy 8 EGF and TGFß mRNA expression 120 Embryo morphology 151

Endocervical pathogens 179 Endometrial cancer 64, 271 
- $\quad$ thickness 64, 113,271

Endometrioma 61

Endometriosis 113,215

-, pathogenesis 261

Endometrium 129

-, mid-cycle 101 ß-Endorphin 42 Endothelin 14 Endothelin-1 190 Estrogens 145 Extrarenal nephroblastoma 141

Female sexuality 217 Fetal blood 227

heart rate 168

markers 231

sex 145

therapy 227

weight estimation 84 Fetoplacental circulation 244 Fibroids 120

Follicle-stimulating hormone 200 Follicular fluid 1

Follow-up 133

Free ß-human chorionic gonadotropm 162

Genital infection 183

- $\quad$ mucosa 97

Glucose 195

Gonadotropin-releasing hormone agonist

117 Granulocyte colony stimulating factor 27

- $\quad$ - - - concentration 5

Granulosa cell 1

Growth hormone 222

Hatching 222 Heart 14 HER-2/neu 209 Heterophile antibodies 139 HIV infection 52 Hormone testing 200

Human chorionic gonadotropin 158

in vitro fertilization 151

papillomavirus 52,265

- DNA 46

perfused cotyledon 244 Hydronephrosis 215

-, pregnancy 274 Hydroureter 215 5-Hydroxytryptamine 244 Hyperplasia 129

Hyperprolactinemia 42 Hypothalamic-pituitary-ovarianaxis, lst-trimesterloss 257

Immunity 133

Immunologic status 52

Implantation, in vitro fertilization 151

Indomethacin 244

Induction 89

-, labor 168,174

In situ hybridization 265

Insulin, women athletes 195

Intrauterine growth retardation 19

- $\quad$ infection 28

In vitro blastocyst development 222

- $\quad$ - fertilization 117

Irradiation 57 
Kidney 14 Koilocytosis 265

Labor 89

Laparoscopy 8

Laser 46

Lithuania 28, 179

Loop electrosurgical excision procedure 46

Low birth weight 183

Lung 14

Luteal phase defect 101

Magnetic resonance angiography 249

- $\quad$ - imaging, insulin response 195

Mouse preimplantation embryos 222

Mozambique 183

Müllerian anomalies, endometriosis 261 Multiple pregnancy, frequency 151 Myometrial resistance vessels 190 Myometrium 11

Natural killer activity 133

- $\quad$ - cell 133

Neutrophil counts, pregnancy 5 Nitric oxide 244

283

Nodes 133

Normal vulvar mucosa 265

Oligosynthetase 46 Ophthalmic artery 32 Ovarian cancer 209

torsion 61

tumors 141 Oxytocin 168,217

Papillomavirus 52 Pathogenesis, endometriosis 261 Pelvic endometriosis 66

- $\quad$ mass 141

Perinatal death 174

PGE release 73

Phosphoglycerokinase 204

Placenta 158

Placental morphometry 253

separation 24

surface area, prediction 253 Polymerase chain reaction 265 Polymorphism 204 Postmenopausal women 271 Prealbumin 231 Preeclampsia 32,244

Pregnancy 36, 80, 145, 158, 190, 244 -, drug-induced agranulocytosis 276

interval 257

rates 101 Pregnant women 5 Preimplantation development, in vitro

fertilization 151

Prelabour rupture of membranes, preterm

period 28 Prematurity 84 Prenatal diagnosis 84, 94, 227 Preoperative oestradiol 125 Preterm

delivery 36

- $\quad$ labour 179

Prolactin 200

Proliferation cell nuclear antigen 129

Prostaglandin E2 89

Pure follicle-stimulating hormone 117 
RU-486 108

SCID mouse 97 Severe preeclampsia 249 Sex behavior 217

- $\quad$ hormones 217

Sonicaid System 8000168

Sonographic echo pattern 113

Spontaneous abortion 36, 257

Steroid hormones 217

Steroid-sensitive proteins 145

Steroidal assays 139

Steroidogenesis 1

Stillbirth 36, 174

T-lymphocytes 133 Third stage of labor 24 Thyroid components 145 Thyroid-stimulating hormone 200 Transabdominal approach 80

- $\quad$ ultrasonography 271

Transferrin 1

Transitional cell carcinoma 278

Transvaginal approach 80

- $\quad$ ultrasonography 271

Tubal coagulation 8

Ultrasonography 80, 84 Ultrasound 101, 108 Ureteral drainage 274

endometriosis 66

obstruction 66 Urethral diverticulum 69 Urinary sample 162 Uterine artery 24, 80

leiomyoma 204

Vaginal cancer 69

operation 125

scan 64 Vasospasm 249 Vulva 46

Vulvar papillomatosis 265

von Willebrand factor 14 Wilm'stumor 141 Women athletes 195

Xenograft 97 X-inactivation 204

284

Subject Index Vol. 40, 1995 